\section{Características antropométricas de nipo-brasileiros}

\section{Anthropometric characteristics of Japanese-Brazilians}

\section{Cana Taniguchi}

Departamento de Medicina Preventiva

Escola Paulista de Medicina - UNIFESP

Rua Botucatu, 740 - Vila Clementino

04023-062 - São PAulo, SP

pitiku@uol.com.br

\section{Suely Godoy Agostinho Gimeno}

Departamento de Medicina Preventiva

Escola Paulista de Medicina - UNIFESP

\section{Sandra Roberta G. Ferreira}

Departamento de Medicina Preventiva

Escola Paulista de Medicina - UNIFESP

\section{Japanese-Brazilian Diabetes Study Group - JBDSG*}

\footnotetext{
* Membros do Japanese-Brazilian Diabetes Study Group - JBDSG: A. Hirai, A.T. Hirai, H.A. Harima K. Osiro, M. lunes (in memoriam), M. Y. Kikuchi, S.R.G. Ferreira, S.G.A. Gimeno (Departamento de Medicina Preventiva - UNIFESP - São Paulo); L.J. Franco (Departamento de Medicina Preventiva da Faculdade de Medicina de Ribeirão Preto - USP); L.K. Matsumura, R.C.S. Moisés (Departamento de Medicina Interna - UNIFESP - São Paulo); M.A. Cardoso (Departamento de Nutrição da Faculdade de Saúde Pública - USP); N. Barros Jr. (Departamento de Cirurgia - UNIFESP - São Paulo); N.E. Tomita (Faculdade de Odontologia de Bauru - USP); K. Wakisaka (Centro de Estudos Nipo-Brasileiros - SP); R.C. Chaim (Faculdade de Nutrição - Universidade do Sagrado Coração Bauru, SP).
}

\section{Resumo}

Objetivo: Este trabalho visou estimar as prevalências de excesso de peso e descrever algumas variáveis antropométricas relativas à distribuição da gordura corporal de uma população migrante de origem japonesa residente no Brasil. Método: A amostra foi constituída por 647 nipo-brasileiros de $1^{\mathrm{a}}(\mathrm{n}=237)$ e $2^{\mathrm{a}}$ geração $(\mathrm{n}=410)$, de ambos os sexos, com idade $\geq 35$ anos, submetidos a medidas antropométricas, de pressão arterial, perfil lipídico e teste oral de tolerância à glicose. Sobrepeso e obesidade foram diagnosticados por meio de índice de massa corporal (IMC) entre $25-29,9 \mathrm{e} \geq 30 \mathrm{~kg} / \mathrm{m}^{2}$, respectivamente. $O$ diagnóstico de adiposidade abdominal foi baseado nos valores de circunferência de cintura $\geq 94 \mathrm{~cm}$ para homens e $\geq 80 \mathrm{~cm}$ para mulheres. Para a análise dos dados foram usados a estatística qui-quadrado e o teste $t$ de Student. Resultados: Dos indivíduos estudados, $40 \%$ apresentavam algum grau de excesso de peso (IMC $\geq 25 \mathrm{~kg} / \mathrm{m}^{2}$ ), sendo a prevalência de obesidade abdominal de $21,5 \%$ entre os homens e de $66,7 \%$ entre as mulheres. Homens nipo-brasileiros $<60$ anos de $2^{\text {a }}$ geração enquadraram-se no perfil andróide de distribuição da gordura corporal; as mulheres mostraram-se mais obesas que os homens, apresentando tanto o padrão andróide como ginóide, avaliado por meio das dobras cutâneas. Conclusão: Os imigrantes japoneses que originalmente não apresentavam o fenótipo de obesidade acompanharam a tendência mundial atual de ganho progressivo de peso até a obesidade. Em particular, essa situação foi acompanhada de aumento de adiposidade abdominal, possivelmente indicativa de acúmulo de gordura visceral e desencadeante de alterações metabólicas. Estes achados sugerem que indivíduos de origem japonesa devem apresentar uma predisposição à obesidade abdominal, que se manifesta quando expostos a ambiente desfavorável.

Palavras-chave: Adiposidade abdominal. Antropometria. Dobras cutâneas. Circunferência da cintura. Doenças metabólicas. Ocidentalização. 
Abstract

Objective: This study aimed to estimate the prevalence of overweight and to describe some indicative anthropometric variables of body fat distribution in a population of Japanese migrants living in Brazil. Methods: The sample consisted of 647 first- $(n=237)$ and second-generation $(n=410)$ Japanese-Brazilians of both genders, aged $\geq 35$ years, who had their anthropometric measures, blood pressure, lipid profile, and oral glucose tolerance test defined. Overweight and obesity were defined as a body mass index (BMI) of $25.0-29.9$ and $\geq 30.0 \mathrm{~kg} / \mathrm{m}^{2}$, respectively; the diagnosis of abdominal adiposity was based on waist circumference values $\geq 94 \mathrm{~cm}$ in men and $\geq 80 \mathrm{~cm}$ in women. Chi-square statistics and Student $t$ test were used for data analysis. Results: Forty percent of the participants showed some degree of overweight $\left(\mathrm{BMI} \geq 25 \mathrm{~kg} / \mathrm{m}^{2}\right.$ ) and the prevalence rates of abdominal adiposity were $21.5 \%$ in men and $66.7 \%$ in women. Second-generation Japanese-Brazilian men, younger than 60 years, had an android profile of fat distribution; women were more obese than men and had both android and gynaecoid patterns, which were assessed by skin fold thickness. Conclusion: Japanese immigrants in Brazil - who originally did not present the obese phenotype - have followed the worldwide trend of progressive weight gain toward the development of obesity. In particular, such situation has been accompanied by an increase in abdominal adiposity, possibly indicating visceral fat accumulation, triggering metabolic disorders. Our findings suggest that Japanese descendants may present predisposition to abdominal obesity, which is triggered when they are exposed to an unfavorable environment.

Key Words: Abdominal adiposity. Anthropometry. Skin fold thickness. Waist circumference. Metabolic disturbances. Westernization.

\section{Introdução}

Em sociedades ocidentais industrializadas como os Estados Unidos e vários países da Europa, a alta prevalência de sobrepeso e obesidade - que ocorre paralelamente ao aumento de doenças crônicas - é considerada o maior problema de saúde pública ${ }^{1}$. Dados antropométricos coletados nos EUA, entre 1988 e 1994, pelo National Health and Nutrition Examination Survey (NHANES III) evidenciaram uma prevalência de obesidade de $19,7 \%$ entre os homens e $24,7 \%$ entre as mulheres [índice de massa corporal (IMC) $\left.>30 \mathrm{~kg} / \mathrm{m}^{2}\right)$ ]. Nos países da Europa que participaram do projeto "Mônica", essa prevalência foi de $15 \%$ entre os homens e $22 \%$ entre as mulheres, observando-se grande variabilidade dos resultados; a prevalência mais alta foi observada na Lituânia, alcançando $32 \%$ entre os homens e $45 \%$ entre as mulheres ${ }^{2}$.

Estudos transversais têm destacado que alguns grupos étnicos têm maior risco que outros para a obesidade, fato que os predispõe a certos tipos de doenças ${ }^{3}$. A obesidade, especialmente a denominada do tipo central, cujo acúmulo de gordura se dá na região abdominal, costuma ser um prenúncio de uma série de conseqüências metabólicas adversas, nas quais se incluem a hiperinsulinemia, tolerância à glicose alterada, hiperlipidemia, além do aumento de tendências trombolíticas e da síndrome plurimetabólica ${ }^{4}$.

O processo de modernização, industrialização e globalização das sociedades tem possibilitado o desenvolvimento de diferentes padrões de vida, que, associados à disponibilidade de serviços e opções de alimentos, favorecem o incremento da prevalência de sobrepeso e obesidade entre os indivíduos.

Estudo efetuado por Franco ${ }^{5}$ destacou que os indivíduos pertencentes à comunidade nipo-brasileira, após sua vinda para o Brasil, passaram a constituir um grupo de alto risco para o diabetes mellitus (DM), provavelmente devido ao processo de ocidentalização, que incluiu mudanças nos hábitos alimentares e nos padrões de atividade física. Ao analisar o aumento de peso desses 
indivíduos, a partir dos 20 anos de idade, Gimeno et al. ${ }^{6}$ mostraram que o risco de desenvolver intolerância à glicose - isolada ou combinada com hipertensão arterial e obesidade abdominal - aumentou em $2 \%$ e $15 \%$, em cada caso respectivamente, por unidade percentual de peso ganho após aquela idade, quando confrontados com indivíduos que permaneceram com peso estável.

Inque e Zimmet $^{7}$ indicaram que, no Japão, houve aumento de mortalidade em pessoas com IMC $\geq 30 \mathrm{~kg} / \mathrm{m}^{2}$. Esses autores, para os quais a classificação do IMC da $\mathrm{WHO}^{2}$ não prediz com precisão a mortalidade multicausal da população na região Ásia-Pacífico, propuseram outra classificação de sobrepeso (IMC $23-24,9 \mathrm{~kg} / \mathrm{m}^{2}$ ), obesidade (IMC $\geq 25 \mathrm{~kg} / \mathrm{m}^{2}$ ) e obesidade central (circunferência de cintura $\geq 90 \mathrm{e} \geq 80 \mathrm{~cm}$, respectivamente, para homens e mulheres) em adultos asiáticos. Ao se avaliar a obesidade, recomendam que a circunferência da cintura seja medida, pois a redução desta, independente da alteração no peso corporal, pode reduzir o risco de doenças.

Em alguns estudos epidemiológicos ${ }^{8,9}, \mathrm{o}$ acúmulo de gordura no tronco foi avaliado por meio da a razão entre dobras cutâneas específicas para verificar associações com doenças cardiovasculares, hipertensão arterial, tolerância diminuída à glicose e outras, observando-se o desenvolvimento de obesidade abdominal nos indivíduos.

Neste trabalho objetivou-se estimar as prevalências de excesso de peso e de obesidade abdominal observadas entre indivíduos de uma comunidade de origem japonesa, participante do estudo conduzido pelo Japanese-Brazilian Diabetes Study Group sobre prevalência de DM e doenças associadas, bem como descrever algumas variáveis antropométricas relativas à distribuição de gordura corporal segundo o sexo, idade e geração.

\section{Material e Métodos}

Os dados são os do estudo de prevalência de DM conduzido entre nipo-brasileiros residentes em Bauru, no Estado de São Paulo.
Em 1988, a população japonesa imigrante no Brasil foi estimada em cerca de 1.228 .000 pessoas, o maior contingente radicado fora do Japão. Desse total, 887.000 indivíduos estabeleceram-se no Estado de São Paulo. Em Bauru, a colonização teve início em 1914, principalmente na área rural. O levantamento demográfico concluído em 1992 mostrou que a população nipo-brasileira residente nesse município totalizava aproximadamente 3.000 indivíduos, sendo $12 \%$ de primeira geração (nascidos no Japão), denominados isseis; $39 \%$ de segunda geração (filhos de, no mínimo, um ascendente de primeira geração), denominados niseis; $30 \%$ de terceira geração denominados sanseis; e 19\% de mestiços (que provêm da miscigenação de raças e gerações).

Os 1.137 residentes na área urbana, com idade $\geq 35$ anos e pertencentes à $1^{\mathrm{a}} \mathrm{e} 2^{\mathrm{a}}$ gerações foram convidados mediante contato telefônico ou visita domiciliar a participar do estudo sobre prevalência de DM e outras doenças associadas, tendo-lhes sido informado o propósito do estudo. A população foi composta pela totalidade dos issei $(\mathrm{n}=$ 284) e por um terço dos indivíduos nisei (amostragem sistemática - da lista ordenada em ordem alfabética, foram selecionados um em cada três), pelo fato de serem mais numerosos, acrescido ainda de $20 \%$ para compensar possíveis perdas $(n=467)$. Dos indivíduos elegíveis para o estudo, não participaram, por falta de resposta ao convite (recusas, mudanças de endereço ou não localização), 15,8\% e 12,6\% dos issei e nisei, respectivamente. A amostra final foi composta por 647 indivíduos, de ambos os sexos. Sete indivíduos foram posteriormente excluídos deste estudo por não apresentarem todas as medidas antropométricas. Participantes e não-participantes não diferiram quanto a sexo e idade.

Os indivíduos selecionados responderam a questionários sobre aspectos relativos à saúde e foram submetidos a exames clínicos (incluindo antropometria e pressão arterial) e de laboratório (foram aferidos os níveis séricos de glicose em jejum [de pelo menos 10 horas] e de 2 horas após sobrecarga com 
75 g de glicose, bem como os de colesterol total e suas frações) no Hospital de Reabilitação de Anomalias Crânio-Facial - USP/ Bauru. O exame clínico completo foi realizado por médicos do Departamento de Medicina Preventiva da UNIFESP-EPM.

Todos os indivíduos que participaram do presente estudo manifestaram seu consentimento, tendo sido também cumpridos os princípios deética da Declaração deHelsinki (1989).

\section{Variáveis antropométricas incluídas no estudo}

- O índice de massa corporal (IMC) foi calculado como peso/estatura ${ }^{2}$, observando as medidas do peso em quilogramas e as da estatura em metros. As medições foram realizadas em balança de consultório com estadiômetro Filizola ${ }^{\circledR}$, utilizando aproximação de 100 g para o peso e de $0,5 \mathrm{~cm}$ para a estatura. Na avaliação, os indivíduos portavam roupas leves e não calçavam sapatos. Procedia-se à calibragem da balança pela manhã, antes do início dos exames. As classificações de sobrepeso e obesidade em adultos observaram as recomendações da $\mathrm{WHO}^{2}$, que estabelece como limites de corte para o IMC os valores entre 18,5$24,9 \mathrm{~kg} / \mathrm{m}^{2}$ (eutrofia), entre $25-29,9 \mathrm{~kg} /$ $\mathrm{m}^{2}$ (sobrepeso) e $\geq 30,0 \mathrm{~kg} / \mathrm{m}^{2}$ (obesidade); para os asiáticos, foram consideradas as de Inque e immet $^{7}$, que propõem valores do IMC entre $18,5-22,9 \mathrm{~kg} / \mathrm{m}^{2}$ (eutrofia), entre $23-24,9 \mathrm{~kg} / \mathrm{m}^{2}$ (sobrepeso) e $\geq 25,0 \mathrm{~kg} / \mathrm{m}^{2}$ (obesidade).

- A circunferência de cintura (CC) foi medida por meio de fita milimetrada flexível, com aproximação de $0,1 \mathrm{~cm}$, em plano horizontal, à altura da cicatriz umbilical, estando o indivíduo ereto, com os braços ao longo do corpo e os pés unidos. A classificação de obesidade central atendeu aos parâmetros da $\mathrm{WHO}^{2}$, que estabelece como ponto de corte para a CC nível 1 valores $\geq 94 \mathrm{~cm}$ para homens e $\geq 80 \mathrm{~cm}$ para mulheres; os valores de $\mathrm{CC} \geq 90 \mathrm{~cm} \mathrm{e} \geq 80 \mathrm{~cm}$ para homens e mulheres, respectivamente, foram aque- les sugeridos para a população asiática ${ }^{7}$.

- As dobras cutâneas abdominal, biciptal, triciptal, subescapular, supra-ilíaca e da coxa foram medidas com o adipômetro Lange ${ }^{\circledR}$, do lado esquerdo do corpo, registrando-se a leitura mais próxima de 0,5 $\mathrm{mm}$ no mostrador. Cada tipo de medida foi obtida em duplicata, calculando-se a média aritmética dos valores. Os pontos anatômicos e a técnica para aplicação das dobras cutâneas tiveram por base as recomendações de Lohman et al. ${ }^{10}$. Foram calculados os somatórios de dobras cutâneas do tronco (subescapular + supra-ilíaca) e dos membros (triciptal + coxa) ${ }^{11}$.

- A razão cintura quadril (RCQ) foi obtida mediante o quociente entre a circunferências da cintura e a do quadril, que é medida no nível da extensão posterior máxima dos glúteos. O ponto de corte utilizado para a obesidade abdominal foi de 1,0 para os homens e 0,85 para as mulheres ${ }^{2}$.

- A porcentagem de gordura corporal (\% GC) foi obtida pela equação específica para japoneses nativos ${ }^{12}$, com base no somatório de duas dobras cutâneas (triciptal e subescapular). Para converter a densidade corporal em \% GC foram aplicadas as fórmulas específicas à etnia ${ }^{13}$, por faixa etária; a estimativa de \% GC foi calculada mediante a fórmula de Brozek et al. ${ }^{14}$. Os valores obtidos de \% GC, correspondentes às situações de risco para os indivíduos, foram comparados com aqueles estabelecidos por Lohman et al. ${ }^{15}$, cujos pontos de corte, no caso de homens e mulheres, são, respectivamente, $\geq 25 \%$ $\mathrm{e} \geq 32 \%$ (risco de doenças associadas ao sobrepeso e à obesidade); entre $16-24 \% \mathrm{e}$ 24-31\% (risco acima da média); $15 \%$ e $23 \%$ (risco médio); entre 6-14\% e 9-22\% (risco abaixo da média); $\mathrm{e} \leq 5 \% \mathrm{e} \leq 8 \%$ (risco de doenças e desordens associadas à desnutrição).

\section{Análise estatística}

Para descrever as características físicas da população foram obtidas medidas de ten- 
dência central e de dispersão, representadas pela média e pelo desvio padrão das variáveis antropométricas. As comparações da freqüência dos indivíduos por idade, geração, IMC, RCQ e CC entre os sexos foram feitas por intermédio da utilização da estatística qui-quadrado. As comparações das médias das variáveis antropométricas entre gerações, sexos e idades foram feitas usando-se o teste $t$ de Student. Os resultados considerados estatisticamente significantes foram aqueles nos quais os valores de $p$ foram menores que 0,05 .

Para os cálculos e a análise estatística foi utilizado o programa de computador STATA (Intercooled) ${ }^{\circledR} 6.0$.

\section{Resultados}

Na Tabela 1 estão apresentados o número e a porcentagem de nipo-brasileiros participantes do estudo segundo o sexo, idade e geração. Houve similaridade na distribuição de grupos $<60$ anos $\mathrm{e} \geq 60$ anos, quanto ao sexo, registrando-se predominância, não significativa, de mulheres da $2^{\text {a }}$ geração em relação às da $1^{\mathrm{a}}$ geração.

Verifica-se, na Tabela 2, o número e a porcentagem de indivíduos segundo o sexo, IMC e presença de obesidade central, de acordo com a classificação da $\mathrm{WHO}^{2}$. Destaca-se que, em ambos os sexos, aproxima- damente $40 \%$ dos indivíduos apresentavam excesso de peso (IMC $\geq 25 \mathrm{~kg} / \mathrm{m}^{2}$ ). Observou-se proporção estatisticamente maior de mulheres com obesidade abdominal, tanto pela CC $(66,7 \%$ vs $21,5 \%$; $\mathrm{p}=0,000)$ como pela RCQ $(76,9 \%$ vs $15,3 \% ; p=0,000)$, quando comparada à dos homens.

Na Tabela 3, a aplicação das categorias do IMC para adultos asiáticos ${ }^{7}$ classificou somente $32,7 \%$ dos nipo-brasileiros como eutróficos, promovendo um substancial aumento na prevalência de indivíduos com sobrepeso e com obesidade, de $40,2 \%$ para $64,5 \%$. A prevalência de obesidade central, medida pela classificação proposta por Inque e Zimmet ${ }^{7}$ para asiáticos, relativa à CC, alterou-se de $21,5 \%$ para $35,5 \%$ entre os homens, porcentagem essa estatisticamente menor que aquela observada entre as mulheres $(66,7 \%$ vs $35,5 \% ; p=0,000)$.

As prevalências de $32,7 \%$ e $57 \%$ de nipobrasileiros eutróficos, baseadas no IMC proposto respectivamente para adultos asiáti$\cos ^{7}$ e ocidentais ${ }^{2}$, diferem daquelas correspondentes à categorização de \% GC, associada ao desenvolvimento de riscos nutricionais $(18,63 \%=15,3 \%+3,3 \%)$. Foi encontrada maior porcentagem de mulheres com alto risco de doenças associadas à obesidade $(52,0 \%)$, quando comparada à dos homens (27,7\%); entretanto, estes apresentaram \% GC aumentada e com risco acima da

Tabela 1 - Distribuição de nipo-brasileiros, segundo a geração, sexo e idade Table 1 - Distribution of Japanese-Brazilians, by generation, gender and age

\begin{tabular}{|c|c|c|c|c|c|c|c|}
\hline \multirow[t]{2}{*}{ Variável } & \multicolumn{2}{|c|}{ Masculino } & \multicolumn{2}{|c|}{$\begin{array}{c}\text { Sexo } \\
\text { Feminino }\end{array}$} & \multicolumn{2}{|c|}{ Total } & \multirow[b]{2}{*}{$p^{*}$} \\
\hline & $\mathrm{N}^{\circ}$ & $\%$ & $\mathrm{~N}^{\circ}$ & $\%$ & No & $\%$ & \\
\hline \multicolumn{8}{|l|}{ Idade * } \\
\hline$<60$ anos & 158 & 51,5 & 173 & 52,0 & 331 & 51,7 & 0,902 \\
\hline$\geq 60$ anos & 149 & 48,5 & 160 & 48,0 & 309 & 48,3 & \\
\hline Total & 307 & 100 & 333 & 100 & 640 & 100 & \\
\hline \multicolumn{8}{|l|}{ Geração } \\
\hline 1. a geração & 124 & 40,4 & 113 & 33,9 & 237 & 37,0 & 0,091 \\
\hline 2. ${ }^{a}$ geração & 183 & 59,6 & 220 & 66,1 & 403 & 63,0 & \\
\hline Total & 307 & 100 & 333 & 100 & 640 & 100 & \\
\hline
\end{tabular}

* Idade média = 58,8 e 58,5 anos para homens e mulheres, respectivamente; mediana = 59 anos para ambos os sexos

* Mean age $=58.8$ and 58.5 years for men and women, respectively; median $=59$ years for both sexes

** Referente à estatística qui-quadrado/ ${ }^{* *}$ Chi-square 
Tabela 2 - Número e porcentagem de nipo-brasileiros segundo o sexo, as categorias do IMC e a obesidade central (CC e RCQ) classificadas pela WHO, 1997

Table 2 - Number and percentage of Japanese-Brazilians according to gender, BMI categories and abdominal obesity (waist circumference and WHR) classified by WHO, 1997

\begin{tabular}{|c|c|c|c|c|c|c|c|}
\hline \multirow{3}{*}{ Variável } & \multirow{2}{*}{\multicolumn{2}{|c|}{ Masculino (307) }} & \multicolumn{2}{|c|}{ Sexo } & \multirow{2}{*}{\multicolumn{2}{|c|}{ Total (640) }} & \multirow[b]{3}{*}{$p^{*}$} \\
\hline & & & Femini & רo $(333)$ & & & \\
\hline & No & $\%$ & $\mathrm{~N}^{\circ}$ & $\%$ & No & $\%$ & \\
\hline \multicolumn{8}{|l|}{ IMC $\left(\mathrm{kg} / \mathrm{m}^{2}\right)^{* *}$} \\
\hline$<18,5$ & 5 & 1,6 & 13 & 3,9 & 18 & 2,8 & 0,359 \\
\hline $18,5-24,9$ & 179 & 58,3 & 185 & 55,7 & 364 & 57,0 & \\
\hline $25-29,9$ & 98 & 31,9 & 108 & 32,6 & 206 & 32,2 & \\
\hline $30-34,9$ & 22 & 7,2 & 25 & 7,5 & 47 & 7,4 & \\
\hline$\geq 35$ & 3 & 1,0 & 1 & 0,3 & 4 & 0,6 & \\
\hline \multicolumn{8}{|c|}{ Presença de obesidade central } \\
\hline \multicolumn{8}{|c|}{ Circunferência de cintura (cm) } \\
\hline $\operatorname{Sim}(C C \geq 94 \text { e } \geq 80)^{* * *}$ & 66 & 21,5 & 222 & 66,7 & 288 & 45,0 & 0,000 \\
\hline Não & 241 & 78,5 & 111 & 33,3 & 352 & 55,0 & \\
\hline \multicolumn{8}{|l|}{ RCQ. } \\
\hline $\operatorname{Sim}(R C Q \geq 1,0 \text { e } \geq 0,85)^{* * *}$ & 47 & 15,3 & 256 & 76,9 & 303 & 47,3 & 0,000 \\
\hline Não & 260 & 84,7 & 77 & 23,1 & 337 & 52,7 & \\
\hline
\end{tabular}

* Referente à estatística qui-quadrado/ ${ }^{*}$ Chi-square

** $\mathrm{n}$ feminino $=332 /{ }^{* *} \mathrm{n}$ women $=332$

*** Circunferência de cintura/RCQ para homens e mulheres, respectivamente/ *** Waist circumference/WHR for men and women, respectively

Tabela 3 - Número e porcentagem de nipo-brasileiros segundo o sexo, o IMC e a obesidade central (CC) para adultos asiáticos ${ }^{7}$, e risco de co-morbidades (\%GC) ${ }^{15}$

Table 3 - Number and percentage of Japanese-Brazilians according to gender, BMI and abdominal obesity (waist circumference) for Asiatic adults ${ }^{7}$, and risk of co-morbidity $(\% B F)^{15}$

\begin{tabular}{|c|c|c|c|c|c|c|c|}
\hline \multirow{3}{*}{ Variável } & \multirow{2}{*}{\multicolumn{2}{|c|}{ Masculino (307) }} & \multirow{2}{*}{\multicolumn{2}{|c|}{$\begin{array}{c}\text { Sexo } \\
\text { Feminino (333) }\end{array}$}} & \multirow{2}{*}{\multicolumn{2}{|c|}{ Total (640) }} & \multirow[b]{3}{*}{$p^{*}$} \\
\hline & & & & & & & \\
\hline & $N^{\circ}$ & $\%$ & No & $\%$ & No & $\%$ & \\
\hline \multicolumn{8}{|l|}{$\mathrm{IMC}\left(\mathrm{kg} / \mathrm{m}^{2}\right)^{* *}$} \\
\hline$<18,5$ & 5 & 1,6 & 13 & 3,9 & 18 & 2,8 & 0,455 \\
\hline $18,5-22,9$ & 106 & 34,5 & 103 & 31,0 & 209 & 32,7 & \\
\hline $23-24,9$ & 73 & 23,8 & 82 & 24,7 & 155 & 24,3 & \\
\hline $25-29,9$ & 98 & 31,9 & 108 & 32,5 & 206 & 32,2 & \\
\hline$\geq 30$ & 25 & 8,2 & 26 & 7,8 & 51 & 8,0 & \\
\hline \multicolumn{8}{|c|}{ Circunferência de cintura (cm) } \\
\hline $\operatorname{Sim}(C C \geq 90 \text { e } \geq 80)^{* * *}$ & 109 & 35,5 & 222 & 66,7 & 331 & 51,7 & 0,000 \\
\hline Não & 198 & 64,5 & 111 & 33,3 & 390 & 48,3 & \\
\hline \multicolumn{8}{|c|}{ Risco de co-morbidades } \\
\hline Abaixo da média**** & 53 & 17,4 & 45 & 13,5 & 98 & 15,3 & 0,000 \\
\hline Médio**** & 10 & 3,2 & 11 & 3,3 & 21 & 3,3 & \\
\hline Acima da média**** & 159 & 51,8 & 104 & 31,2 & 263 & 41,1 & \\
\hline Alto & 85 & 27,7 & 173 & 52,0 & 258 & 40,3 & \\
\hline
\end{tabular}

*Referente à estatística qui-quadrado/ *Chi-square

**n feminino $=332 /{ }^{*} n$ women $=332$

****ircunferência de cintura para homens e mulheres, respectivamente/ *** Waist circumference for men and women, respectively

****De doenças associadas à obesidade ${ }^{* * * *}$ Risk of illness associated with obesity 
média (51,8\%), na mesma proporção de alto risco para doenças associadas à obesidade das mulheres. Ambas as diferenças foram estatisticamente significantes $(p<0,000)$.

As informações sobre as variáveis antropométricas estão apontadas na Tabela 4 , com seus valores de média e de desviopadrão, segundo o sexo. Não houve diferenças estatisticamente significantes entre os sexos, em relação aos valores médios de IMC. Foram observados valores maiores nas medidas que avaliam a gordura corporal entre as mulheres (todas as dobras cutâneas, \% GC estimada pela equação específica de predição para a população japonesa e somatório de dobras cutâneas dos membros). Os resultados das medidas que avaliam a distribuição de gordura corporal, isto é, RCQ, CC e soma das dobras cutâneas do tronco, mostraram-se altos entre os homens, quando comparados aos das mulheres. Os valores da RCQ e da CC não representaram risco de doenças para os homens, mas sim para as mulheres, pois, com base na classificação da $\mathrm{WHO}^{2}$, os valores 0,90 e 83,82 cm sugeriram alto risco de morbidade associado a esses indicadores.

Ao examinar as diferenças dos valores médios das variáveis antropométricas entre as mulheres (Tabela 5) observa-se que as de $2^{\text {a }}$ geração e $<60$ anos, quando comparadas às de $1^{\text {a }}$ geração $\mathrm{e} \geq 60$ anos, apresentaram os valores mais elevados entre todas as medidas estudadas, exceto a CC e a RCQ. Esses valores, que representam a obesidade central, foram mais altos entre as mulheres de $1^{\text {a }}$ geração. No caso dos homens, as diferenças dos valores médios das variáveis antropométricas foram mais elevadas entre os de

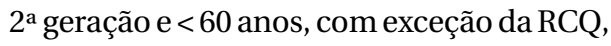
para os quais não foram observadas diferenças estatisticamente significantes, quando comparados aos de $1^{\text {a }}$ geração.

\section{Discussão}

Os resultados do presente trabalho indicaram altas prevalências de sobrepeso e obesidade entre os nipo-brasileiros de meia-idade, reproduzindo uma tendência que se dissemina mundialmente na atualidade. Cabe ressaltar que esse grupo populacional, etnicamente homogêneo, não apresentava o fenótipo da obesidade. $\mathrm{O}$ excesso de peso (no caso dos homens) e a obesidade (no caso das mulheres) observados foram ressalta-

Tabela 4 - Características antropométricas (valores de média e desvio padrão) de nipo-brasileiros, segundo o sexo Table 4 - Anthropometric characteristics of Japanese-Brazilians (mean and standard deviation values), according to gender

\begin{tabular}{|c|c|c|c|c|c|}
\hline \multirow{3}{*}{ Variável } & \multicolumn{4}{|c|}{ Sexo } & \multirow[b]{3}{*}{$p^{*}$} \\
\hline & \multicolumn{2}{|c|}{ Masculino $(n=307)$} & \multicolumn{2}{|c|}{ Feminino $(n=333)$} & \\
\hline & Média & D.P. & Média & D.P. & \\
\hline Índice de massa corporal $\left(\mathrm{kg} / \mathrm{m}^{2}\right)$ & 24,46 & 3,64 & $* * * 24,41$ & 3,61 & 0,442 \\
\hline Dobra cutânea do antebraço (mm) & 7,90 & 3,68 & $* * * 10,51$ & 4,46 & 0,000 \\
\hline Dobra cutânea do bíceps (mm) & 6,99 & 3,65 & 12,48 & 5,89 & 0,000 \\
\hline Dobra cutânea do tríceps (mm) & 13,26 & 6,02 & 23,00 & 7,50 & 0,000 \\
\hline Dobra cutânea subescapular (mm) & 22,27 & 9,83 & 27,51 & 10,17 & 0,000 \\
\hline Dobra cutânea do tórax (mm) & 22,62 & 9,97 & - & - & - \\
\hline Dobra cutânea abdominal (mm) & 27,93 & 10,48 & $* * 35,72$ & 10,91 & 0,000 \\
\hline Dobra cutânea da antecoxa (mm) & 16,30 & 7,68 & 28,29 & 10,97 & 0,000 \\
\hline Dobra cutânea supra-ilíaca (mm) & 21,33 & 9,79 & 25,13 & 9,79 & 0,000 \\
\hline Gordura corporal (\%) & 21,12 & 7,16 & 32,91 & 9,60 & 0,000 \\
\hline Circunferência de cintura $(\mathrm{cm})$ & 87,96 & 8,80 & 83,82 & 9,87 & 0,000 \\
\hline Razão cintura quadril & 0,95 & 0,06 & $* * * 0,90$ & 0,07 & 0,000 \\
\hline Soma de dobras do tronco (mm) & 66,86 & 24,95 & $* * * * 63,93$ & 19,23 & 0,048 \\
\hline Soma de dobras de membros (mm) & 56,85 & 23,03 & 75,64 & 24,59 & 0,000 \\
\hline
\end{tabular}

* Referente ao teste $t$ de Student/ ${ }^{*}$ Student t test

** $\mathrm{n}$ feminino $=330 /{ }^{* *} n$ women $=330$

$*^{* *}=332 /{ }^{* * * *}=329$ 
Tabela 5 - Diferenças entre os valores médios das variáveis antropométricas e valor de p (test $t$ de Student) segundo o sexo, geração e idade

Table 5 - Differences among means values of anthropometric variables and $p$ value (Student $t$ test) according to gender, generation and age

\begin{tabular}{|c|c|c|c|c|}
\hline \multirow{3}{*}{ Variável } & \multicolumn{4}{|c|}{ Sexo } \\
\hline & Masculino & Feminino & Masculino & Feminino \\
\hline & \multicolumn{2}{|c|}{$\left(1^{\mathrm{a}} \times 2^{\mathrm{a}}\right.$ geração $)$} & \multicolumn{2}{|c|}{$(<60 \times 360$ anos $)$} \\
\hline & \multicolumn{4}{|c|}{$t$ de Student $(p)$} \\
\hline Índice de massa corporal $\left(\mathrm{kg} / \mathrm{m}^{2}\right)$ & $-1,65(0,000)$ & $-0,46(0,137)$ & $1,54(0,000)$ & $0,27(0,249)$ \\
\hline Dobra cutânea do antebraço $(\mathrm{mm})$ & $-1,59(0,000)$ & $-2,11(0,000)$ & $1,65(0,000)$ & $2,51(0,000)$ \\
\hline Dobra cutânea do bíceps (mm) & $-2,07(0,000)$ & $-2,45(0,000)$ & $2,14(0,000)$ & $2,83(0,000)$ \\
\hline Dobra cutânea do tríceps (mm) & $-2,44(0,000)$ & $-3,18(0,000)$ & $2,67(0,000)$ & $2,93(0,000)$ \\
\hline Dobra cutânea subescapular (mm) & $-6,35(0,000)$ & $-4,82(0,000)$ & $5,13(0,000)$ & $4,79(0,000)$ \\
\hline Dobra cutânea do tórax (mm) & $-4,42(0,000)$ & - & $3,69(0,001)$ & - \\
\hline Dobra cutânea abdominal (mm) & $-5,68(0,000)$ & $-3,45(0,003)$ & $5,96(0,000)$ & $4,05(0,000)$ \\
\hline Dobra cutânea da antecoxa (mm) & $-2,56(0,002)$ & $-5,61(0,000)$ & $3,34(0,000)$ & $7,92(0,000)$ \\
\hline Dobra cutânea supra-ilíaca (mm) & $-6,00(0,000)$ & $-4,52(0,000)$ & $6,43(0,000)$ & $5,03(0,000)$ \\
\hline Gordura corporal (\%) & $-4,28(0,000)$ & $-4,67(0,000)$ & $3,79(0,000)$ & $4,48(0,000)$ \\
\hline Circunferência de cintura $(\mathrm{cm})$ & $-2,83(0,003)$ & $0,56(0,313)$ & $2,11(0,018)$ & $-1,13(0,148)$ \\
\hline Razão cintura quadril & $0,01(0,196)$ & $0,02(0,015)$ & $-0,01(0,088)$ & $-0,02(0,002)$ \\
\hline Soma de dobras do tronco $(\mathrm{mm})$ & $-12,67(0,000)$ & $-9,27(0,000)$ & $12,99(0,000)$ & $12,10(0,000)$ \\
\hline Soma de dobras de membros (mm) & $-14,80(0,000)$ & $-12,51(0,000)$ & $14,23(0,000)$ & $12,75(0,000)$ \\
\hline
\end{tabular}

dos, particularmente, pela porcentagem de indivíduos com adiposidade central, obtida por indicadores antropométricos que estimam a distribuição de gordura corporal.

Nos estudos prévios ${ }^{6,16,17}$ sobre a comunidade nipo-brasileira de Bauru, há dados que confirmam o alto risco desses indivíduos para doenças como a tolerância à glicose alterada, DM, hipertensão arterial e dislipidemia. Tais doenças estão associadas ao excesso de peso e à obesidade central, avaliados pelo IMC, RCQ e CC.

Aplicando-se a classificação do IMC para adultos asiáticos, a população em estudo apresentou $24,3 \%$ de indivíduos com sobrepeso (IMC $23-24,9 \mathrm{~kg} / \mathrm{m}^{2}$ ), 32,2\% com obesidade tipo I (IMC $25-29,9 \mathrm{~kg} / \mathrm{m}^{2}$ ) e $8 \%$ com obesidade tipo II (IMC $\geq 30 \mathrm{~kg} / \mathrm{m}^{2}$ ). Esses resultados se aproximam das prevalências observadas entre os norte-americanos, de acordo com os dados coletados entre $1988 \mathrm{e}$ 1994 pelo National Health and Nutrition Survey-NHANES III, que apontou $20 \%$ dos homens e $25 \%$ das mulheres como obesos (IMC $\geq 30 \mathrm{~kg} / \mathrm{m}^{2}$ ), todos na faixa etária entre 20-74 anos. Essas prevalências de obesida- de, comparadas às obtidas por meio da categorização do IMC para adultos asiáticos, corresponderiam aos indivíduos com sobrepeso, no caso da população de Bauru. Nesse sentido, $32,1 \%$ dessa população com IMC $25-29,9 \mathrm{~kg} / \mathrm{m}^{2}$ seriam considerados obesos; tais porcentagens são bastante elevadas e próximas às dos países da Polinésia (Samoa), onde se verificam as maiores prevalências mundiais de obesidade ${ }^{2}$.

Estudos ${ }^{18,19}$ apontam que as prevalências de obesidade e de sobrepeso, relativamente maiores em populações migrantes, podem ser reflexo do impacto da ocidentalização no estilo de vida e na dieta desses grupos étnicos ao se fixarem em outros países. Neste contexto, as prevalências de obesidade tipo I e II, assim como as de adiposidade central encontradas na comunidade nipo-brasileira, podem, presumivelmente, ser resultado do processo de aculturação em apenas duas gerações ${ }^{5}$.

Os indivíduos de $2^{\text {a }}$ geração cuja idade era inferior a 60 anos apresentaram \% GC mais elevada em comparação aos de $1^{\text {a }}$ geração mais idosos ( $\geq 60$ anos), dado que é 
estatisticamente significante. Alterações importantes poderiam estar ocorrendo na proporção de gordura corporal dos nipo-brasileiros avaliados, fato que coincide com as considerações de Kuczmarski ${ }^{20}$. Segundo este autor, a gordura do corpo aumenta paulatinamente entre 25-45 anos, havendo para ambos os sexos, um contínuo acúmulo de massa gorda até os 70-75 anos, e um posterior declínio.

Outra característica relevante seria a de que, devido à extensão mais curta dos membros inferiores em relação à altura do tron$\mathrm{CO}^{21} \mathrm{e}$ à mesoformidade no somatotipo ${ }^{22} \mathrm{da}$ população japonesa, os nikkei são mais baixos, atarracados e mais musculosos que os caucasianos; conseqüentemente, a obtenção de valores mais elevados do IMC para esses indivíduos seria equivalente aos de massa gordurosa. Por outro lado, há evidências de que a relação entre \% GC e IMC varia conforme a etnia da população estudada: os asiáticos possuem maior grau de adiposidade para um determinado IMC, comparado ao da população branca ${ }^{23}$. Apesar de os nipobrasileiros geralmente não apresentarem obesidade (IMC $\geq 30 \mathrm{~kg} / \mathrm{m}^{2}$ ), essa característica pareceu afetar todas as mulheres, visto que a média dos valores de \% GC foi de $35,06 \%$ e de $30,58 \%$ para aquelas com idades $<60$ anos e $\geq 60$ anos, respectivamente. Dados semelhantes foram observados no Japão $0^{24}$ em mulheres de meia-idade, entre 39 e 48 anos (36\% GC), e idosas, entre 61 e 78 anos (31\% GC).

Stevens et al. ${ }^{25}$ assinalaram que os índices que medem rudimentarmente a gordura corporal e a obesidade abdominal, como o IMC, a RCQ e a CC, podem falhar na identificação de indivíduos com risco de doenças, não predizendo com precisão a morbimortalidade em grupos étnicos. No presente estudo, a proporção total de nikkei sob risco de doenças associadas à obesidade (40,3\%), aproxima-se de $40,2 \%$, ou seja, daquela obtida pela categorização do IMC para adultos asiáticos (IMC $\geq 25 \mathrm{~kg} / \mathrm{m}^{2}$ ), proposta por Inque e Zimmet ${ }^{7}$.

$\mathrm{Na}$ avaliação dos nipo-brasileiros de Bauru, as medidas de espessura das dobras cutâneas, consideradas separadamente, forneceram informações quanto à distribuição relativa da gordura subcutânea de cada região do corpo, mas não podem predizer, isoladas, e de forma acurada, a gordura corporal tota ${ }^{26}$. Nesse aspecto, os valores médios de todas as dobras cutâneas relacionadas à gordura subcutânea apresentaram-se maiores nas mulheres, quando comparados aos dos homens. Para estes, configurou-se a obesidade do tipo central ou andróide (formato de maçã), com acúmulo nítido de gordura nas regiões superiores do corpo: abdome, tronco, cintura, escápula e tórax. As medidas de dobras cutâneas verificadas entre as mulheres sugeriram a obesidade ginóide ou inferior (formato de pêra), com acúmulo de gordura na parte inferior do corpo - quadril e coxa anterior; entretanto, por haver ainda ocorrido deposição de gordura na região abdominal, caracterizou-se também o perfil andróide nas mulheres. A adiposidade periférica e a do tronco foram confirmadas pelo somatório de dobras cutâneas de membros e tronco, especialmente entre as mulheres de $2^{\text {a }}$ geração e com idade $<60$ anos.

Os valores médios das dobras cutâneas biciptal, triciptal, subescapular e abdominal obtidos entre os nikkei corroboram os achados de Wang et al. ${ }^{19}$, pois estes autores, ao testarem a validade das equações de Durnin e Womersley e de Jackson para asiáticos com idade entre 18 e 98 anos, observaram que as medidas de dobras cutâneas do bíceps, do abdome, supra-ilíaca e subescapular eram significativamente superiores, quando comparadas às da população branca. Entre as mulheres, os valores das dobras cutâneas subescapular e abdominal sobressaíram na comparação com outros estudos, porém houve concordância na evidência de que os compartimentos intra-abdominais aumentam com a progressão da idade ${ }^{27}$. Observouse, outrossim, similaridade na disposição adiposa corporal entre os nikkeis e os mexicano-americanos, que possuem gordura subcutânea troncular maior que a dos brancos, mas com distribuição diferenciada ${ }^{28}$.

Um estudo prospectivo para verificar o efeito da idade, do sexo e da alteração no 
peso sobre o modelo de distribuição corporal, medido pela RCQ, observou que a circunferência do quadril diminui em mulheres de meia idade. Nos homens, a CC normalmente aumenta e, quando há ganho de peso, a razão entre as duas circunferências torna-se maior que a RCQ inicial. Nas mulheres, a CC e a RCQ variam proporcionalmente aos valores originais ${ }^{29}$.

Com base nas características de obesidade central características dos membros da comunidade nipo-brasileira de Bauru, foi possível inferir que a presença da adiposidade visceral seria um motivo presumível de alterações metabólicas. Esta suposição fundamentou-se em estudos efetuados por pesquisadores ${ }^{18,30}$ para os quais a obesidade abdominal mostrou associação com a resistência insulínica e com a gênese do DM. Tais resultados podem estar ainda relacionados às variações étnicas da obesidade central nas diferentes populações estudadas.

De forma geral, as medidas de dobras cutâneas complementam as avaliações antropométricas clínicas convencionais na predição de tolerância à glicose ${ }^{31}$. A medição do tecido subcutâneo tem sido freqüentemente utilizada por profissionais de educação física, e em programas de saúde pública, devido às dificuldades metodológicas em se viabilizar a utilização de aparelhos mais onerosos; além disso, pode fornecer a estimativa da \% GC.

Neste trabalho, algumas comparações dos valores de circunferências e de espessura de dobras cutâneas com aqueles obtidos por outros especialistas nacionais e internacionais ficaram prejudicadas pelo fato de a metodologia aplicada ter sido diversa e, principalmente, devido à não existência de homogeneidade entre as faixas etárias estudadas. As divergências aqui verificadas em relação às medidas antropométricas, especialmente das mulheres, sugerem o fato de que tanto as dimensões corporais quanto a distribuição da gordura subcutânea podemse modificar em vários aspectos, sobretudo se considerados diferentes países e etnias.

\section{Referências}

1. Kopelman PG, Finer N, Fox KR, Hill A, MacDonald IA. ASO consensus statement on obesity. Int J Obes 1994; 18: $189-91$.

2. World Health Organization. Preventing and managing the global epidemic of obesity. [Report of the World Health Organization Consultation of Obesity]. Geneva; 1997.

3. Hill JO, Sidney S, Lewis CE, Tolan K, Scherzinger AL, Stamm ER. Racial differences in amounts of visceral adipose tissue in young adults: the CARDIA (Coronary Artery Risk Development in Young Adults) Study. Am J Clin Nutr 1999; 69: 381-7.

4. Dudeja V, Misra A, Pandey RM, Devina G, Kumar G, Vikram NK. BMI does not accurately predict overweight in Asian Indians in northern India. Br J Nut 2001; 86: 105-12.

5. Franco LJ. Diabetes in Japanese-Brazilians - influence of the acculturation process. Diab Res Clin Pract 1996; 34: S51-S57.

6. Gimeno SGA, Ferreira SRG, Cardoso MA, Franco LJ, Iunes M, and Japanese-Brazilian Diabetes Study Group. Weight gain in adulthood and risk of developing glucose tolerance disturbance: a study of a Japanese-Brazilian population. J Epidemiol 2000; 10: 103-10.
7. Inoue S, Zimmet P. The Asia-Pacific perspective: Redefining obesity and its treatment. WHO Western Pacific Region. Australia; 2000.

8. Couillard C, Lemieux S, Moorjani S, Lupien PJ, Thériault G, Prud'Homme D et al. Associations between 12-year changes in body fatness and lipoprotein-lipid levels in men and women of the Québec Family Study. Int J Obes 1996; 20: 1081-8.

9. Katzmarzyk PT, Malina RM, Song TMK, Bouchard C. Physique, subcutaneous fat, adipose tissue distribution, and risk factors in the Québec Family Study. Int J Obes 1999; 23: 476-84.

10. Lohman TG, Roche AF, Martorell R. Anthropometric standardization reference manual. Champaign, Illinois: Human Kinetics Books; 1988.

11. Costa RF. Um estudo epidemiológico. In: Composição corporal - teoria e prática da avaliação. $1^{\text {a }}$ ed. São Paulo; Manole; 2001. p. 65-97.

12. Nagamine S, Suzuki S. Anthropometry and body composition of Japanese young men and women. Human Biol 1964; 36: 8-15. 
13. Heyward VH, Stolarczyk LM. Composição corporal e etnias. In: Avaliação da composição corporal aplicada. São Paulo: Manole; 2000. p. 138-142.

14. Brozek J, Grande F, Anderson JT, Keys A. Densitometric analysis of body composition: revision of some quantitative assumptions. Ann New York Acad Sci 1963; 110:113-40.

15. Lohman TG. Advances in body composition assessment. Current issues in exercise science series. Monograph no 3. Champaign, Illinois: Human Kinetics Books; 1992.

16. Ferreira SRG, Iunes M, Franco LJ, Iochida LC, Hirai A, Vivolo MA, and Japanese-Brazilian Diabetes Study Group. Disturbance of glucose and lipid metabolism in first and second generation of Japanese-Brazilians. Diab Res Clin Pract 1996; 34: 59-63.

17. Lerario DDG, Gimeno SGA, Franco LJ, Iunes M, Ferreira SRG e Grupo de Estudos do Diabetes na Comunidade Nipo-Brasileira, São Paulo, SP, Brasil. Excesso de peso e gordura abdominal para síndrome metabólica em nipo-brasileiros. Rev Saúde Pública 2002; 36: 4-11.

18. Fujimoto WY, Newell-Morris LL, Grote M, Bergström RW, Shuman WP. Visceral fat and morbidity: NIDDM and atherogenic risk in Japanese men and women. Int J Obes 1991; 15:41-4.

19. Wang J, Thornton JC, Russell M, Burastero S, Heymsfield SB, Pierson Jr RN. Asians have lower body mass index (BMI) but higher percent body than do whites: comparisons of anthropometric measurements. Am J Clin Nutr 1994; 60: 23-8.

20. Kuczmarski RJ. Need for body composition information in elderly subjects. Am J Clin Nut 1989; 50: 1150-7.

21. Greulich WW. A comparison of the physical growth and development of American born and native Japanese children. Am J Phys Anthropol 1957; 19: 173-84.

22. Heath BH, Hopkins CE, Miller CD. Physiques of Hawaii-born young men and women of Japanese ancestry, compared with college men and women of United States and England. Am J Phys Anthropol 1961; 19: $173-84$.
23. Deurenberg P, Yap M, Van Staveren WA. Body mass index and percent body fat: a meta analysis among different ethnic groups. Int J Obes Relat Metab Disord 1998; 22: 1164-71.

24. Tsunenari T, Tsutsumi M, Ohno K, Yamamoto Y, Kawaktsu M, Shimogak K et al.. Age - and gender related changes in body composition in Japanese subjects. J Bone Miner Res 1993; 8: 297-402.

25. Stevens J, Keil JE, Rust PF, Tyroler HA, Davis CE, Gazes PC. Body mass index and body girth as predictors of mortality in black and white women. Arch Int Med 1992; 152: 1257-62.

26. Borkan GA, Helts DE, Gerzof SG, Robbins AH. Comparison of body composition in middle-aged and elderly males using computer tomography. Am J Phys Anthropol 1985; 66: 289-95.

27. Martin A, Drinkwater DT. Variability in the measures of body fat. Sport Med 1991; 11: 277-88.

28. Wei M, Gaskill SP, Haffner SM, Stern MP. Waist circumference as the best predictor of noninsulin dependent diabetes mellitus compared to body mass index, waist/hip ratio and other anthropometric measurements in Mexican Americans - a 7 year prospective study. Obes Res 1997; 5: 16-23.

29. Shimokata H, Andres R, Coon PJ, Elahi D, Muller DC, Tobin JD. Studies in the distribution of body fat. II Longitudinal effects of change in weight. Int $J$ Obes 1989; 13: 455-64.

30. Nagaretani H, Nakamura T, Funahashi T, Kotani K, Miyanaga $\mathrm{M}$, Tokunaga $\mathrm{K}$ et al. Visceral fat is a major contributor for multiple risk factor clustering in Japanese men with impaired glucose tolerance. Diabetes Care 2001; 24: 2127-33.

31. Sievenpiper JL, Jenkins DJA, Josse RG, Leiter LA, Vuksan V. Simple skinfold measurements complement conventional anthropometric assessments in predicting glucose tolerance. Am J Clin Nutr 2001; 73: 567-73.

recebido em: 28/06/2004

versão final apresentada em: 23/11/2004 aprovado em: 13/12/2004 